Hydroécol. Appl. (1995) Tome 7 Vol. 1-2, pp. 1-18

\title{
L'utilisation de substrats artificiels pour l'analyse biologique comparée des lacs. Durée d'exposition
}

\section{Comparative analysis of the bottom fauna of lakes by the mean of artificial substrates. Exposure time}

\author{
H. Gimaret ${ }^{(1)}$ \\ (1) Laboratoire d'Hydrobiologie-Hydroécologie - Institut des Sciences et Techniques de l'Envi- \\ ronnement (ISTE) de I'Université de Franche-Comté - Place Leclerc, 25030 Besançon cedex.
}

Résumé. - Des substrats artificiels ont été posés en zones littorale et profonde du lac de Saint-Point et relevés après 2, 4 et 8 semaines d'exposition.

Le temps d'exposition pour une colonisation quantitative et qualitative optimale se situe autour de 4 semaines.

La similarité calculée au moyen du coefficient semi-métrique de Sorensen, entre les peuplements de substrats (surface prospectée : $0,12 \mathrm{~m}^{2}$ ) et celui récolté au moyen d'un protocole standard d'échantillonnage du sédiment meuble (surface prospectée : $0,84 \mathrm{~m}^{2}$ ) est globalement faible. II s'avère que cette différence n'est pas imputable à la variété taxonomique, mais à la densité et surtout à la nature de la faune. Les différences observées s'accentuent avec la profondeur.

$\|$ reste à définir le nombre de substrats nécessaire et suffisant à l'obtention d'un échantillon relativement stable des systèmes considérés.

L'influence de la nature du substrat conduit à rechercher une méthode d'évaluation de la qualité des eaux lacustres fondée sur l'utilisation de substrats artificiels standards mis en place à différentes profondeurs.

Mots clés. - Lacs - Macrobenthos - Substrats artificiels.

Abstract. - Artificials substrates have been immerged in the littoral and profundal zones of Saint-Point lake (Jura) and sampled with an exposure time of 2, 4 and 8 weeks.

The optimal quantitative and qualitative colonization is obtained with a time exposure of 4 weeks.

The similarity, calculated with the Sørensen's coefficient, between the littoral bottom fauna collected in artificial substrates (prospected area: $0,12 \mathrm{~m}^{2}$ ) and the one collected by a standard protocol of the light sediment (prospected area : $0,84 \mathrm{~m}^{2}$ ) is low. It seems that 
this difference is not imputable to the taxonomic diversity, but to the density and principally to the taxa's nature. The differences become more pronounced in the profundal zone. It remains to define the adequate number of substrates in order to obtain a representative sampling of a lake system. The influence of substrate's nature upon the composition of the benthic populations induce to search a method of lacustrine water quality evaluation based on the utilisation of standard artificial substrates exposing in different depths.

Key Words. - Lakes - Bottom fauna - Artificial substrates.

\section{INTRODUCTION}

Un des problèmes majeurs rencontrés dans l'analyse de la structure biologique des peuplements benthiques en milieu lacustre est l'échantillonnage du substrat sédimentaire. II faut en effet définir un protocole qui permette l'analyse comparée des systèmes. Dans cette perspective, Verneaux et al, (1993) ont proposé un protocole d'échantillonnage dans lequel le nombre d'échantillons minimal par isobathe est fonction de l'importance du plan d'eau et de la profondeur prospectée. Un tel protocole, appliqué au lac de Saint Point, d'une superficie de 403 ha, implique le prélèvement de 21 échantillons de sédiments en zone littorale $(-1$ à $-2 \mathrm{~m})$. A la charge de travail de collecte des échantillons (une journée pour 3 personnes) s'ajoute le temps requis pour le tri des échantillons: 10 journées pour une personne.

Indépendamment de la lourdeur de l'échantillonnage sédimentaire, cette méthode se heurte dans certains cas à la difficulté de trouver des sédiments fins aux faibles profondeurs. La pente peut également être trop forte pour permettre un échantillonnage aux profondeurs stipulées dans le protocole.

L'emploi de substrats artificiels (Laville 1974) est susceptible de pallier ces inconvénients. Afin de préciser les modalités de colonisation d'un type de substrat, une étude a été réalisée sur le lac de Saint-Point (massif du Jura).

Les listes faunistiques établies sur les échantillons provenant des substrats artificiels ont été comparées aux relevés réalisés sur des sédiments prélevés à la benne en vue de définir le temps d'exposition optimal des substrats.

\section{TECHNIQUE UTILISÉE}

\subsection{Les substrats artificiels}

II est possible de distinguer quatre groupes de substrats artificiels:

- les paniers à substrats minéraux ou synthétiques (Moon 1935 et 1940, Wene \& Wicliff 1940, Britt 1955, Scott 1958, Kajak 1964, Henson 1965, Anderson \& Mason 1968, Ulfstrand 1974, Fullner 1971, Jacobi 1971, Dickson \& Cairns 1972, Mason \& al., 1973, Benfield \& al. 1974, La- 
ville op. cit., Khalaf 1975, Bournaud \& Cellot 1981, Cellot 1982);

- les substrats imitant des structures végétales (bryophytiques le plus souvent) (Egglishaw 1964, Glime \& Clemons 1971, Macan \& Kitching 1972);

- les substrats "multiplaques" (Hester \& Dendy 1962, Arthur \& Horning 1969, Sents \& Braconnot 1966, Fullner, op. cit., Mason \& al. op. cit., Mac Daniel 1974, Malesieux, 1976, Verneaux \& al. 1977);

- les systèmes pour les milieux hyporhéiques (Schwoerbel 1967, Coleman \& Hynes, 1970, Bishop 1972, Hynes 1975).

\subsection{Matẻriel employé (fig. 1)}

Les substrats utilisés dans cette étude, sont du type "multiplaques", ils constituent une version simplifiée de ceux proposés par Verneaux et al. (1977) pour la prospection des eaux courantes. Si la version originale était composée de trois niveaux, la version employée n'en comporte plus que deux (fig. 1).

- le niveau inférieur comporte une distribution régulière de volumes vides formés, d'une part, par des couloirs concentriques séparant 3 rangées de cubes et, d'autre part, des espaces libres alternant avec les cubes d'une même rangée. La section des galeries ainsi constituées diminuant vers le centre suivant une progression géométrique de raison 2 et la sole de ce labyrinthe présente une granulométrie grossière, sélectionnée

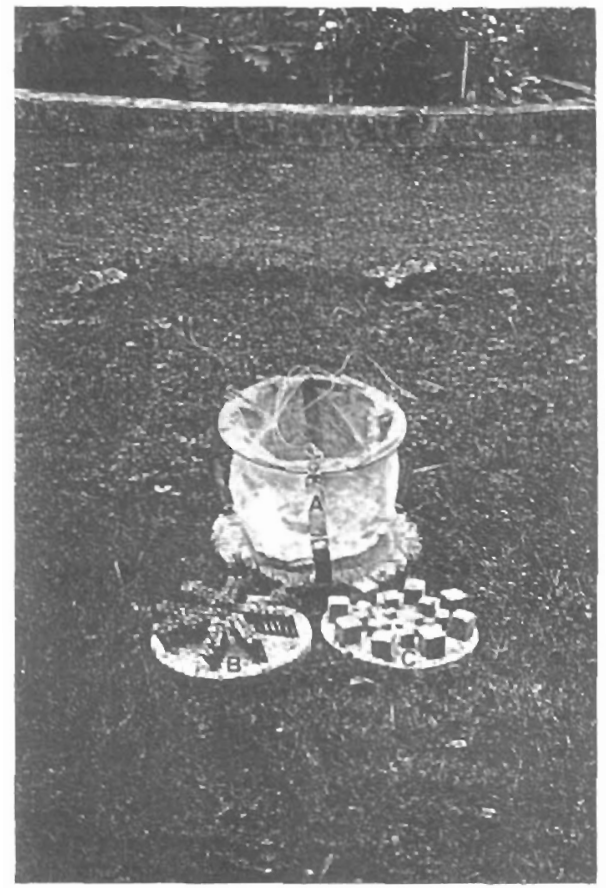

Fig. 1. - substrat artificiel démonté :

A : support-piège en position de relevage

$B$ : niveau supérieur

$C$ : niveau inférieur.

Fig. 1. - artificial substrate dismantled.

A : "Trap-support" in raising position

B: upper level

$\mathrm{G}$ : inferior level.

au tamis et comprise entre 1,25 et $1,50 \mathrm{~mm}$;

- le niveau supérieur est constitué d'un disque de ciment dans lequel sont scellées des brosses simularit une végétation aquatique.

Ces deux parties sont fixées sur un support-piège composé d'une large semelle circulaire en acier inoxydable au milieu de laquelle est fixé un axe vertical permettant le centrage des dif- 
férents niveaux et l'adaptation du crochet de manœuvre. Un filet de toile repose sur cette plaque et sa partie supérieure est fixée sur une couronne qui, en se relevant, empêche la fuite des organismes lors de la récupération des supports. Cette particularité constitue un élément essentiel du dispositif destiné à recueillir des données quantitatives.

Un substrat représente une surface de prélèvement de $0,06 \mathrm{~m}^{2}$ tandis qu'un prélèvement de sédiments constitué de deux bennes Eckman modifiée par station - représente une surface de $0,04 \mathrm{~m}^{2}$. La comparaison sera effectuée à partir des densités rapportées au $1 / 10 \mathrm{~m}^{2}$.

\subsection{Localisation des stations - Périodes d'exposition}

Le lac de Saint-Point est le plus vaste des lacs naturels du Jura franccomtois et s'inscrit, à $850 \mathrm{~m}$ d'altitude, dans le paysage de la haute vallée du Doubs, dominé par les pâturages et les sapinières.

Le lac, traversé par le Doubs, occupe une dépression synclinale où les formations du Crétacé (Albo-Cénomanien) sont surmontées de placages glaciaires (fig. 2a); à la fonte du glacier würmien, les moraines ont assuré la fermeture de la cuvette en créant un unique plan d'eau; les lacs de Saint-Point et de Remoray ont été

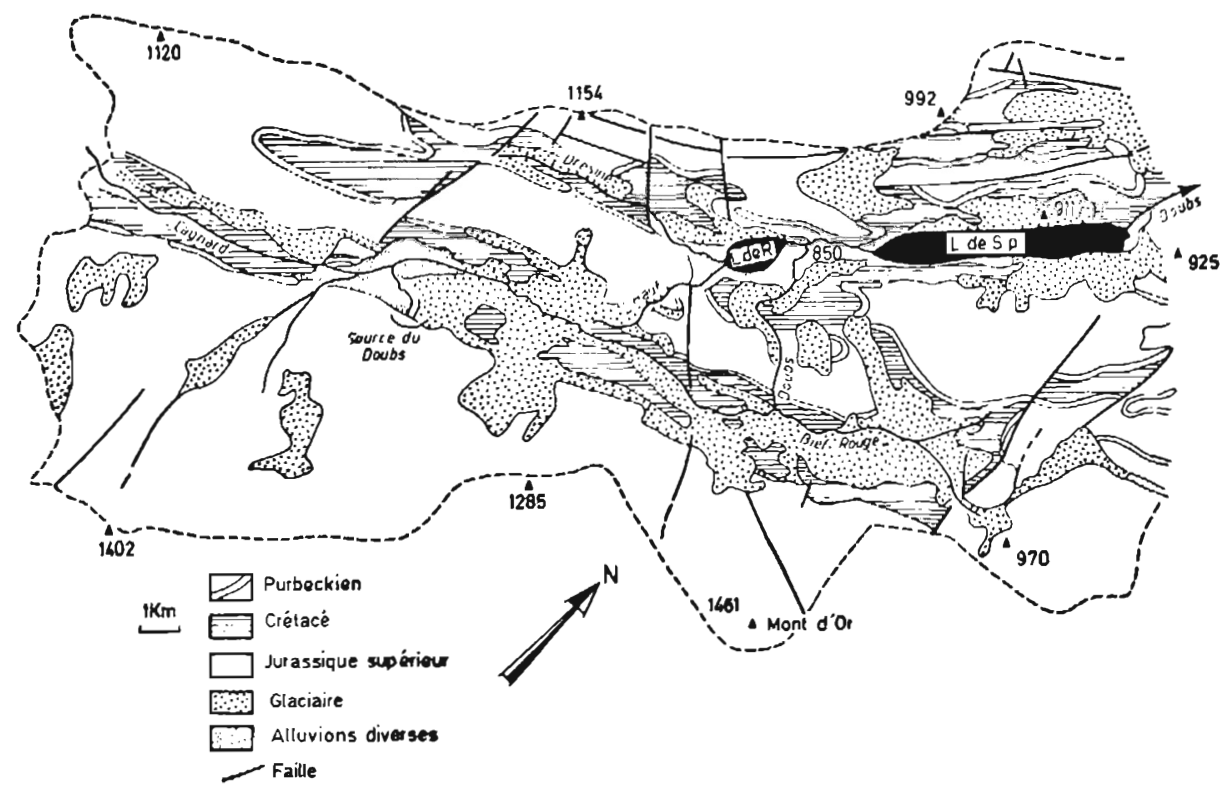

Fig. 2a. - Géologie du bassin versant du lac de Saint-point.

Fig. 2a. - Geology of Saint-Point lake's catchment basin. 
séparés ultérieurement par accumulation des alluvions du Doubs.

\section{- Stations de la zone littorale (fig. 2b)}

Deux stations ont été placées en zone littorale du lac de Saint-Point en fonction de la pente de la beine. Ainsi, la station 1 est située sur une beine de faible largeur et à pente forte, tandis que la station 2 se situe sur une beine plus longue à pente faible. Trois substrats ont été posés à chaque station le 11 mai 1990. Un support a été relevé sur chaque station au bout de 2, 4 et 8 semaines d'exposition.

\section{- Station de la zone limnétique (fig. 2b)}

Après analyse des résultats obtenus en zone littorale, deux supports ont été posés le 5 octobre 1990 et exposés pendant 4 semaines sur un point à -30 mètres (station 3) déjà échantillonné au printemps 1990 avec une benne du type Eckman modifiée, afin de tester l'efficacité des substrats artificiels en profondeur.

\subsection{Exploitation et traitement des données}

L'analyse comparative des peuplements va s'effectuer sur les variables que constituent la variété taxonomique et la densité, au moyen du coefficient de similarité de Sørensen (in Legendre \& Legendre 1979).

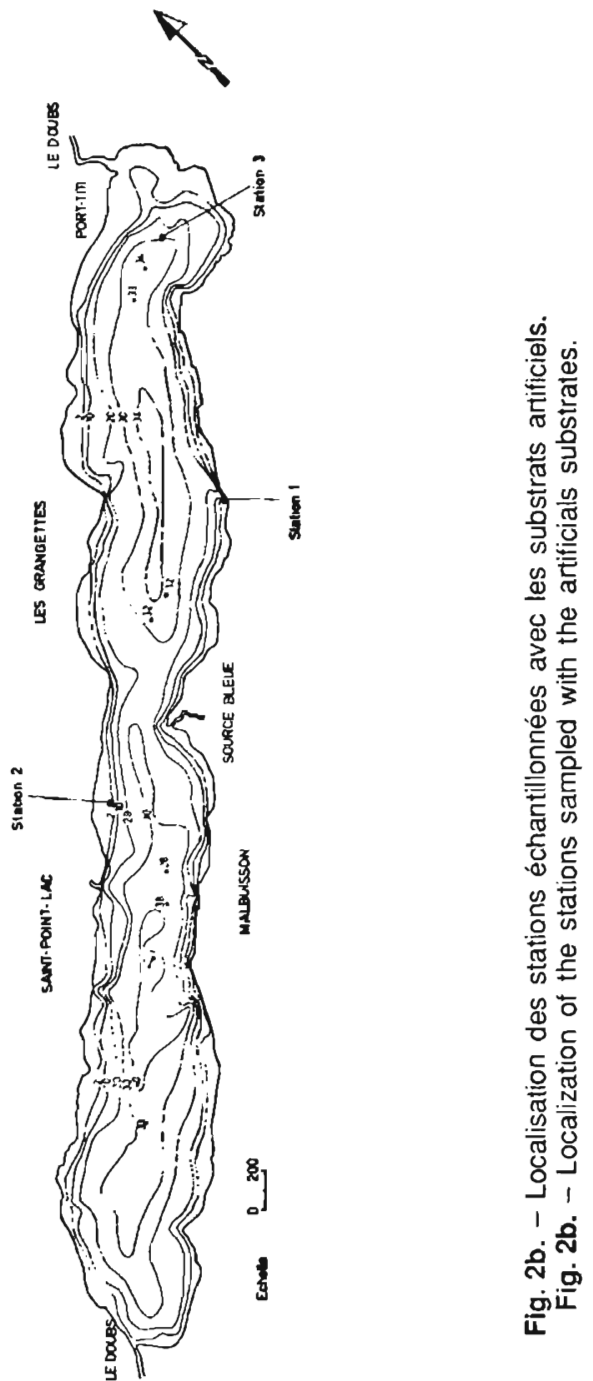


3 RÉSULTATS

\subsection{Répertoires faunistiques} (tab. I et II)

\section{a) zone littorale}

Le tableau I présente la liste des taxons de la zone littorale répertoriés à l'aide des substrats artificiels (en fonction du temps d'exposition) et de la benne.

\section{b) zone limnétique}

Le tableau II présente la liste des taxons de la zone profonde $(-30 \mathrm{~m})$ inventoriés à l'aide des substrats artificiels et de la benne.

\subsection{Temps d'exposition}

\subsubsection{Variété taxonomique}

La figure 3 présente l'évolution de la variété taxonomique des stations 1 et 2 cumulées.

Quelle que soit la station considérée, la variété taxonomique est maximale après 4 semaines d'exposition et semble se stabiliser voire légèrement régresser ensuite confirmant les données de Moon (op. cit.). D'après Laville (op. cit.) la durée d'exposition nécessaire serait double (2 mois) dans les lacs pyrénéens.

En zone littorale, ont été recensés, sur $0,12 \mathrm{~m}^{2}$ de substrat $\left(2 \times 0,06 \mathrm{~m}^{2}\right)$, 45 taxons, contre 46 avec 21 stations et $0,84 \mathrm{~m}^{2}$ prospecté avec la benne, et 19 contre 15 en zone profonde avec 12 stations et $0,48 \mathrm{~m}^{2}$.

\subsubsection{Densité}

La figure 4 présente l'évolution de la densité globale des stations 1 et 2 cumulées.

Dans tous les cas, la densité est maximale après 4 semaines d'exposition et régresse de façon importante ensuite.

Au regard de ces premiers résultats, il apparaît que la durée d'exposition optimale se situe autour de 4 semaines.

\subsubsection{Modification des peuplements}

a) Evolution de la densité (fig. 5a): la densité est maximale après 4 semaines d'exposition pour la majorité des catégories de taxons à l'exception des Ephéméroptères, des Chironomini et des Mollusques.

b) Evolution de la variété (fig. 5b) : le nombre de taxons par catégorie est maximal au bout de 4 semaines d'exposition sauf pour les Trichoptères, les Mollusques et les taxons divers.

\subsection{Comparaison des peuplements "substrats" - "sédiments"}

\subsubsection{Similarites}

Le tableau III présente les résultats de l'application du coefficient de similarité de Sørensen (in Legendre et Legendre, op. cit.) appliqué aux deux listes faunistiques de la zone littorale et de la zone profonde. 
Tableau I. - Répertoire et densité en ind $/ 0,1 \mathrm{~m}^{2}$ des taxons inventoriés en zone littorale à l'aide de la benne et des substrats artificiels au lac de St Point.

$2 s$, $4 \mathrm{~s}$ et $8 \mathrm{~s}$ : durée d'exposition en semaines.

Les taxons soulignés sont propres aux substrats.

Table I. - Index and density in ind. $/ 0.1 \mathrm{~m}^{2}$ of the taxa inventoried with the grab and the artificial substrates in the littoral zone of St. Point lake.

2s, $4 \mathrm{~s}$ and $8 \mathrm{~s}$ : time exposure in weeks.

The underlined taxons are specific to the substrates.

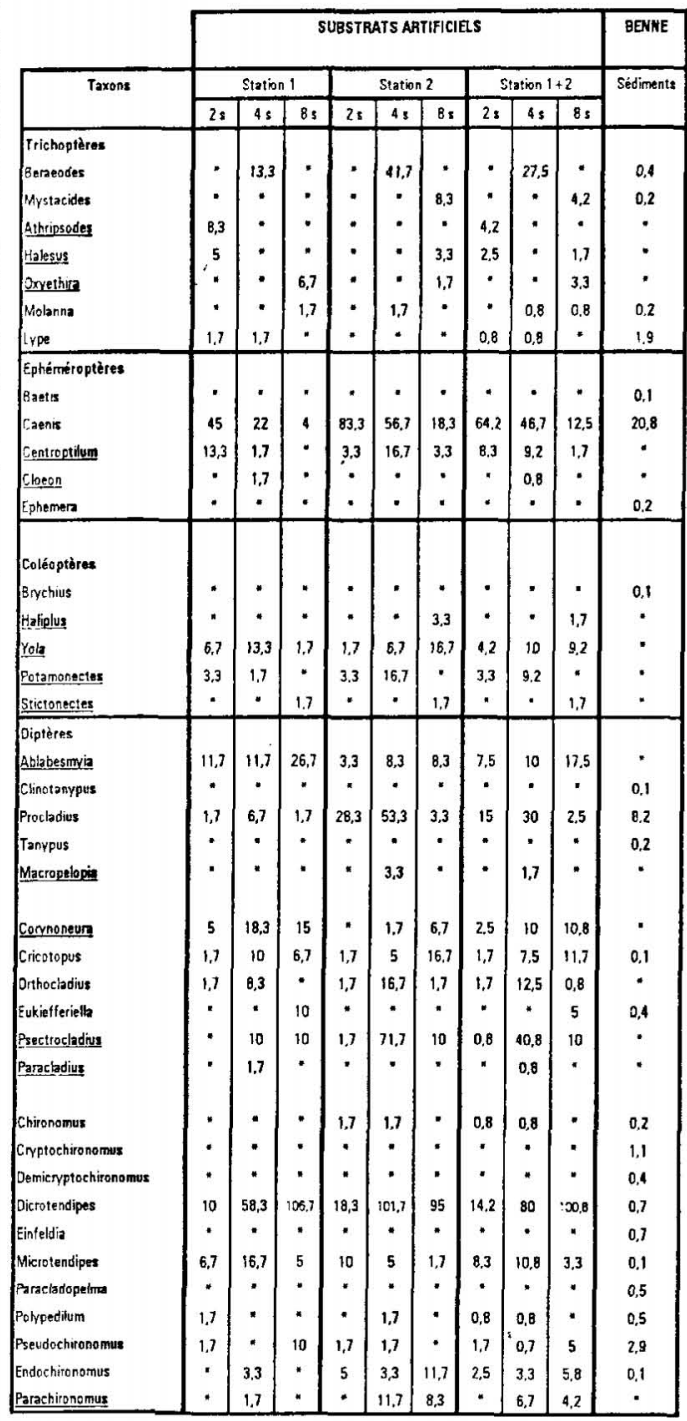

\begin{tabular}{|c|c|c|c|c|c|c|c|c|c|c|}
\hline \multirow[t]{2}{*}{ Taxong } & \multicolumn{3}{|c|}{ Station 1} & \multicolumn{3}{|c|}{ Station 2} & \multicolumn{3}{|c|}{ Station $1+2$} & \multirow[t]{2}{*}{ Sédiments } \\
\hline & 25 & 45 & 83 & 28 & $4 s$ & $8 s$ & 25 & 45 & $B_{5}$ & \\
\hline Cladotanytarsus & 3.3 & 63,3 & 21,7 & 50 & 465 & 51.7 & 267 & 264,2 & 36,7 & 11,7 \\
\hline Paratanytarzus & 20 & 458,3 & 200,3 & 33,3 & 181,7 & 50 & 26,7 & 325 & $: 26.7$ & 0.2 \\
\hline Tanytarsus & 6.7 & 86.7 & 91,7 & 20 & 273.3 & 28,3 & 13.3 & 180 & 60 & 2 \\
\hline Ceratopogonisan & 5 & 5 & 3.3 & 8.3 & 11,7 & $\cdot$ & 6,1 & 8,3 & 1.7 & 14,2 \\
\hline Chaoborus & $\therefore$ & $\cdot$ & - & - & $\cdot$ & - & • & $\cdot$ & " & 0.4 \\
\hline Helius & - & - & - & - & - & - & * & * & - & 0,1 \\
\hline Tabanus & - & 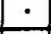 & $\therefore$ & $\dot{.}$ & $\dot{-}$ & $\dot{-}$ & $\therefore$ & $\cdot$ & - & 0.2 \\
\hline $\begin{array}{l}\text { Odonstex } \\
\text { Coenagrion }\end{array}$ & - & - & - & 3.3 & - & . & 1.7 & * & . & . \\
\hline $\begin{array}{l}\text { Mégnloptèrex } \\
\text { Sialis }\end{array}$ & & & & & & & & & & \\
\hline Sialis & $\dot{-}$ & $\dot{-}$ & 1,7 & $\therefore$ & 1,7 & i & 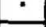 & 0,8 & 0,8 & 1,3 \\
\hline Crustacés & & & & & & & & & & \\
\hline Asedus & 11.7 & 6.7 & 71,7 & " & 11,7 & ${ }^{60}$ & 5,8 & 9,2 & 65,8 & $\dot{0}$ \\
\hline Gammarus & 6.7 & 8,3 & 5 & 6.7 & 15 & 23,3 & 6.7 & 11,7 & 16.2 & 0.1 \\
\hline Oiconecles & $\cdot$ & $\dot{ }$ & $\dot{-}$ & $\dot{ }$ & 1.7 & $\therefore$ & $\cdot$ & 0.8 & $\therefore$ & $\therefore$ \\
\hline La mellibry anches & & & & & & & & & & \\
\hline Pisidium & $\cdot$ & 5 & 5 & 3.3 & 3.3 & 5 & 1.7 & 4.2 & 5 & 7.6 \\
\hline Spharium & $\cdot$ & $\dot{ }$ & : & $\cdot$ & 1,7 & 5 & $\cdot$ & 0,8 & 2.5 & 0.5 \\
\hline Gastéropodes & & & & & & & & & & \\
\hline Bithynia & 20 & 13,3 & 28,3 & • & • & • & 10 & 6,7 & 14.2 & 0.4 \\
\hline Bythinelta & $\cdot$ & $\cdot$ & ' & • & $\cdot$ & - & $"$ & $\cdot$ & $\cdot$ & 0.2 \\
\hline Vatrata & - & • & 8.3 & " & 5 & 10 & $\cdot$ & 2.5 & 9,2 & 0.6 \\
\hline Pranorbis & - & $\cdot$ & $\cdot$ & $\cdot$ & - & $\cdot$ & - & $\cdot$ & • & 0.2 \\
\hline Gyzuluz & • & - & $\cdot$ & • & $\cdot$ & 1.7 & $\cdot$ & $\cdot$ & 0.8 & $\cdot$ \\
\hline Radx & - & - & - & 1.7 & - & - & 0.8 & " & $\cdot$ & - \\
\hline Myxas & $\dot{ }$ & $\cdot$ & $\dot{1}$ & $\dot{ }$ & $\dot{ }$ & 30 & 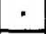 & $\cdot$ & 15 & $\cdot$ \\
\hline Achètes & & & & & & & & & & \\
\hline Erpobdelela & 15 & 5 & 16,7 & • & " & 5 & 7,5 & 2.5 & $: 0,8$ & 1.7 \\
\hline Grossiphona & 1,7 & $\cdot$ & 1.7 & $\cdot$ & $\cdot$ & $\cdot$ & 0.8 & $\cdot$ & 0.8 & 0.7 \\
\hline Helobdellita & 5 & 16.7 & 6.7 & $\cdot$ & • & 15 & 2,5 & 8.3 & 10,8 & 0.7 \\
\hline Piscicola & $\cdot$ & $\cdot$ & 1.7 & 1.7 & 1.7 & $\cdot$ & 0.8 & 0.8 & 0.8 & $\dot{-}$ \\
\hline Pianaires & & & & & & & & & & \\
\hline Dendrocoelum & $\cdot$ & 3.3 & 5 & - & 1.7 & - & · & 2.5 & 2.5 & - \\
\hline Qugasia & 38,3 & 5 & 10 & 5 & 11,7 & 3.3 & 21,7 & 8,3 & 6.7 & $\dot{ }$ \\
\hline Oligochetes & & & & & & & & & & \\
\hline Tubificicida à..s.c. & 1.7 & 18,3 & 20 & 1,7 & 15 & 11.7 & 1.7 & 16,7 & 15,8 & 7.1 \\
\hline Tubificidae s.s.c. & - & 28,3 & 35 & - & 1.7 & $\cdot$ & - & 15 & 17.5 & 10 \\
\hline Naididae & 16.7 & 38,3 & 40 & 16,7 & 83,3 & 85 & 16,7 & 60.8 & 62.5 & - \\
\hline Cnidaires & & & & & & & & & & \\
\hline Hydia & 26.7 & 33,3 & 16,7 & 18,3 & 50 & 16,7 & 22,5 & 41,7 & 9.7 & . \\
\hline Hydracariens & 83,3 & 50 & 25 & 16.7 & 33.3 & 25 & 50 & 41,7 & 25 & $\therefore$ \\
\hline Nómatodes & $\cdot$ & $\dot{\circ}$ & $\dot{ }$ & $\cdot$ & $\cdot$ & $\cdot$ & $\dot{ }$ & $\cdot$ & $\cdot$ & 0.6 \\
\hline Variét & 31 & 35 & 35 & 28 & 39 & 35 & 37 & 45 & 45 & 46 \\
\hline
\end{tabular}


Tableau II. - Répertoire et densité en ind. $/ 0,1 \mathrm{~m}^{2}$ des taxons inventoriés en zone profonde $(-30 \mathrm{~m})$ à l'aide de la benne et des substrats artificiels au lac de St. Point.

Table II. - Index and density in ind. $/ 0.1 \mathrm{~m}^{2}$ of the taxa inventoried with the grab and the artificial substrates in the profundal zone $(-30 \mathrm{~m})$ of St. Point lake.

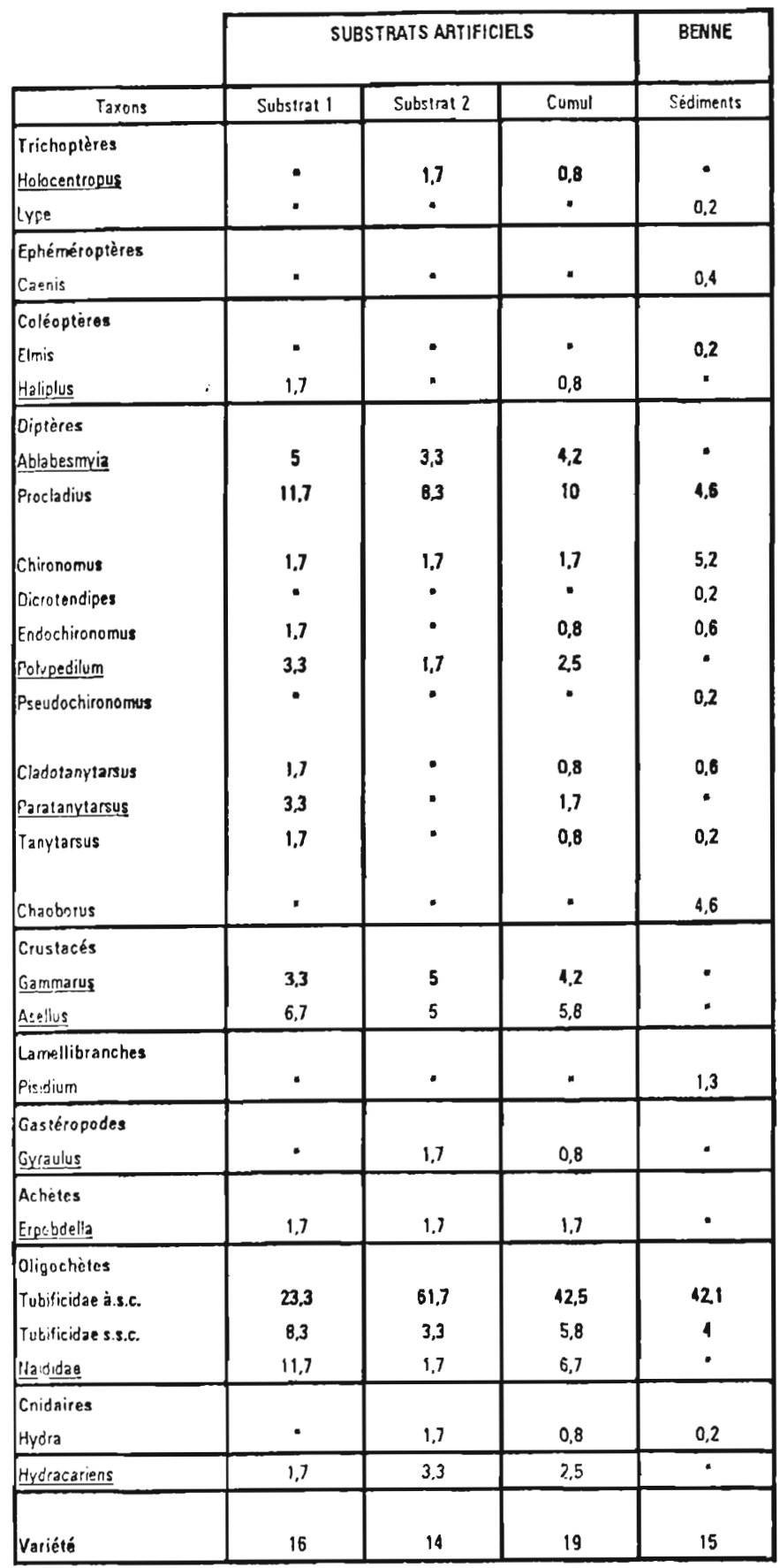




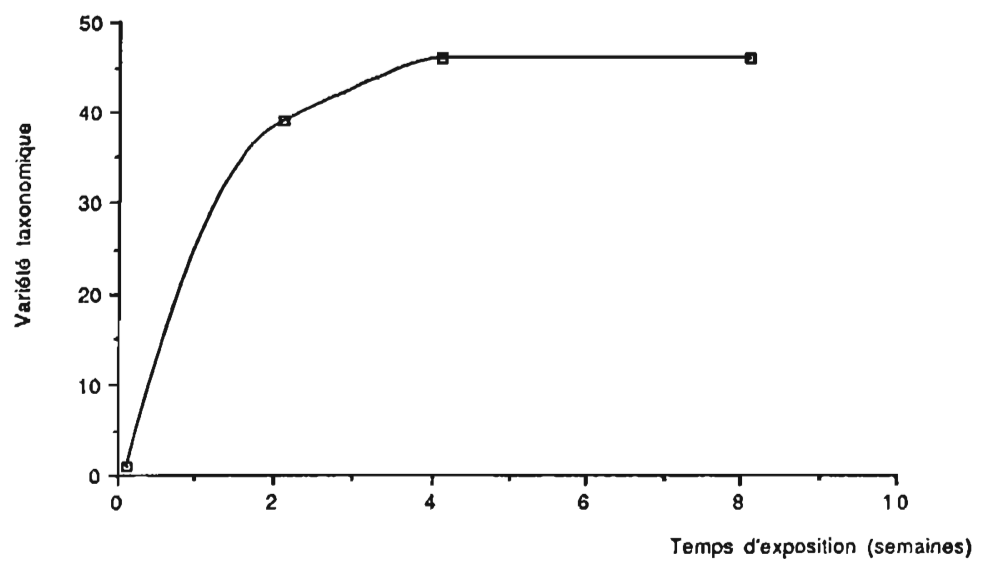

Fig. 3. - Évolution de la variété taxonomique en fonction du temps d'exposition.

Fig. 3. - Evolution of the taxonomic richness in relation with the time exposure.

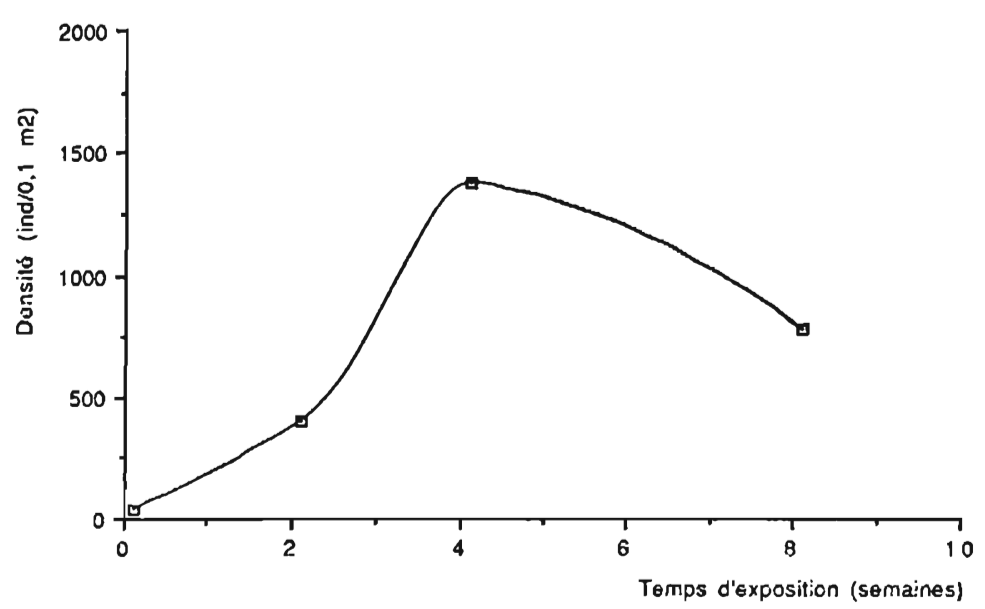

Fig. 4. - Évolution de la densité en fonction du temps d'exposition.

Fig. 4. - Evolution of the density in relation with the time exposure.

Globalement les valeurs du coefficient de similarité sont faibles quelle que soit la profondeur considérée $(0,58$ en zone littorale et 0,47 en zone limnétique). La similarité est toutefois maximale en zone littorale après une durée d'exposition de 4 semaines. Cette seule durée d'exposition sera prise en compte pour l'analyse suivante.

Cette faible similarité peut s'analyser au travers de trois paramètres: la 

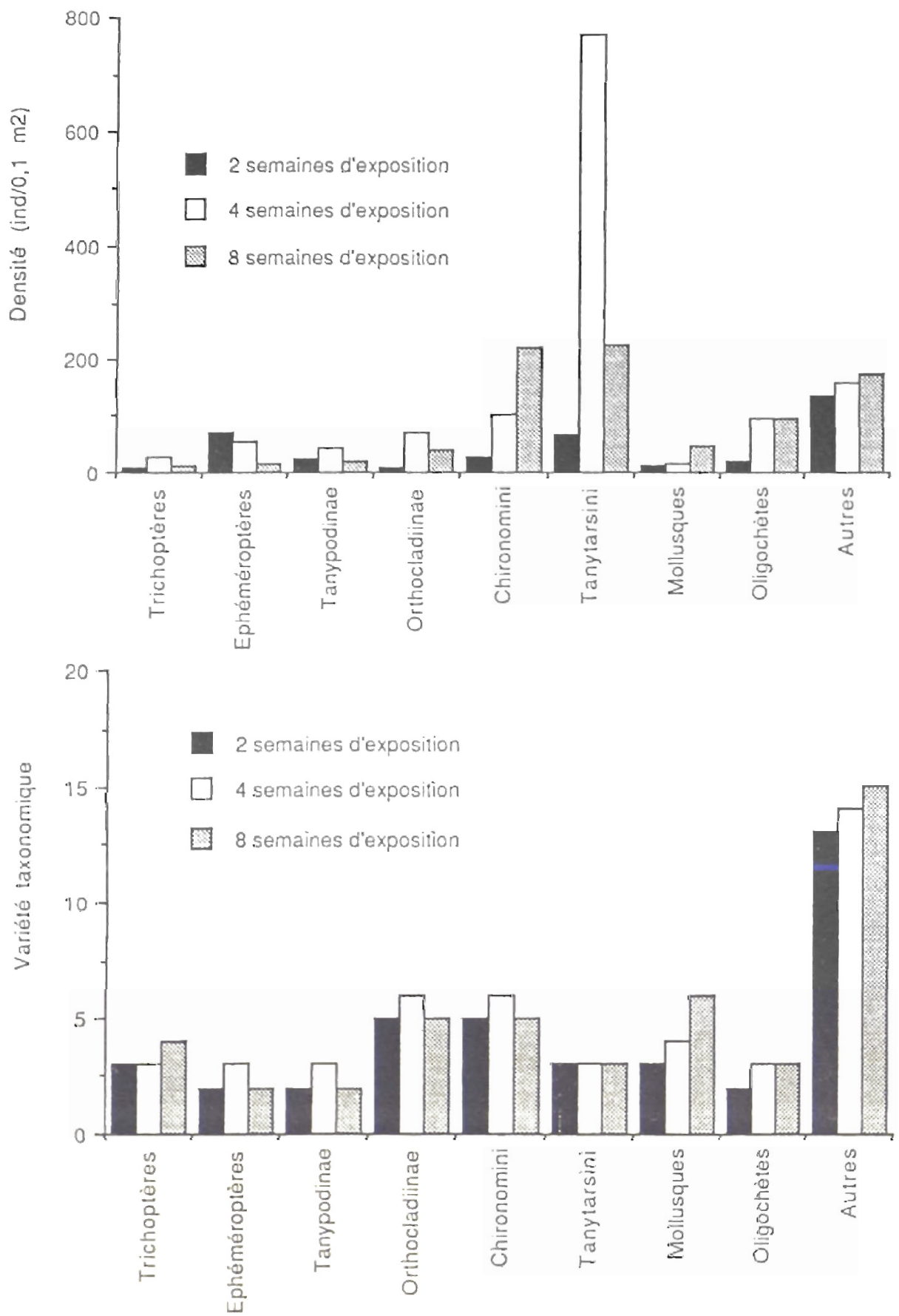

Fig. 5 a et b. - Modification quantitative et qualitative des peuplements en fonction de la durée d'exposition.

Fig. 5a et b. - Quantitative and qualitative modification of population in relation with the time exposure. 
Tableau III. - Calcul des coefficients de similarité entre les peuplements récoltés à l'aide la benne et des substrats artificiels.

Table III. - Coefficient of similarity calculation between the populations inventoried with the grab and the artificial substrates.

\begin{tabular}{|l|c|c|c|c|}
\hline \multicolumn{1}{|c|}{ S8 } & 7one litt. / $\mathrm{scm}$. & 7one litt. / 4 sem. & Zone litt. / 8 sem. & Zonc prof. / 4 sem. \\
\hline Trichoptèrcs & & & & 0 \\
\hline Ephéméroptèrcs & 0,29 & 0,86 & 0,5 & 0 \\
\hline Tanypodinac & 0,4 & 0,33 & 0,4 & 0,67 \\
\hline Orthocladiinac & 0,33 & 0,33 & 0,4 & $*$ \\
\hline Chironomini & 0,75 & 0,29 & 0,57 & 0,57 \\
\hline Tanytarsini & 1 & 0,71 & 0,53 & 0,8 \\
\hline Mullusques & 0,44 & 1 & 1 & 0 \\
\hline Oligochìtcs & 0,5 & 0,8 & 0,67 & 0,8 \\
\hline Autres & 0,42 & 0,67 & 0,67 & 0,22 \\
\hline & & 0,4 & 0,46 & 0,47 \\
\hline
\end{tabular}

variété taxonomique, la nature de la faune et la densité.

\subsubsection{Variété taxonomique}

- Zone littorale: 45 taxons ont été répertoriés avec les substrats artificiels et 46 dans les sédiments, prélevés in situ.

- Zone limnétique: 19 taxons ont été répertoriés avec les substrats artificiels et 15 dans les sédiments, prélevés in situ.

La faible similarité constatée en zone littorale ne peut être expliquée par la différence de la variété taxonomique (1 seul taxon)

En zone profonde, la différence atteint $21 \%$ (quatre taxons de plus sur les substrats) et peut déjà expliquer le faible coefficient de similarité observé. A cette profondeur, compte tenu que l'habitat est uniquement constitué par du sédiment meuble, il est normal de constater que l'attractivité du substrat artificiel est beaucoup plus nette qu'en zone littorale dont la mosaïque habitationnelle est plus complète.

\subsubsection{Nature de la faune}

\section{a) zone littorale}

Sur les 63 taxons recensés par les deux échantillonnages, 18 sont spécifiques aux sédiments, 19 sont spécifiques aux substrats et 26 sont communs.

La figure 6 présente les coefficients de similarité calculés par catégories de taxons et classés par ordre décroissant.

II apparaît que les catégories discriminantes sont les Orthocladiinae, 


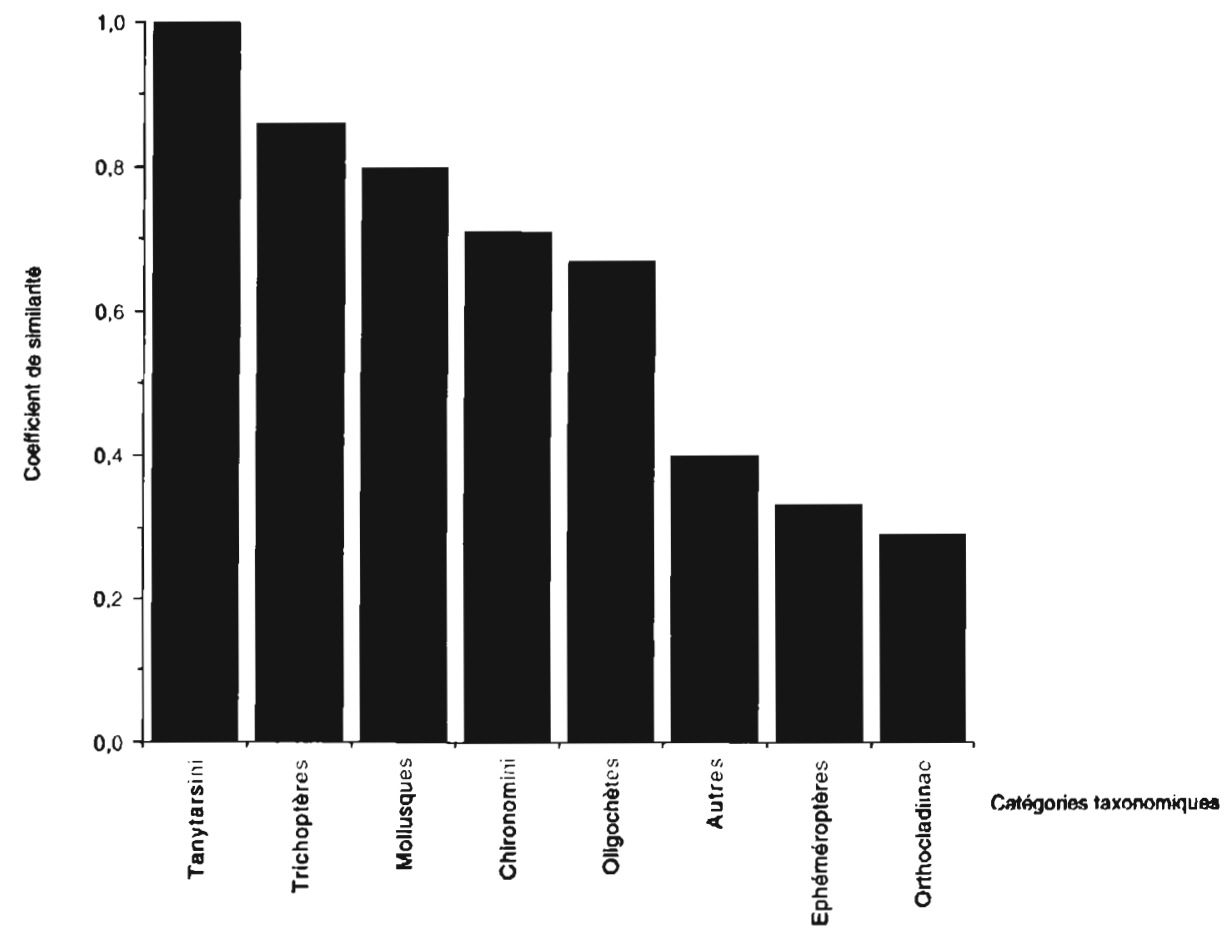

Fig. 6. - Coefficient de similarité calculé par catégorie de taxons : classement par ordre décroissant. Fig. 6. - Calculated similarity's coefficient in relation to the taxa's categories.

les Tanypodinae, les Ephéméroptères et les taxons divers.

Les Orthocladiinae présentent davantage d'affinité pour les substrats que pour les sédiments $(5$ genres dans les substrats artificiels contre 2 dans les sédiments et 1 seul commun).

Les Ephéméroptères et les Tanypodinae possèdent deux genres spécifiques aux substrats artificiels et aux sédiments et un seul commun.

En ce qui concerne les taxons divers, les substrats hébergent prioritairement les taxons épibenthiques
(Cnidaires, Hydracariens, Planaires, Coléoptères aux stades adulte et larvaire), tandis que les sédiments hébergent principalement des organismes endobenthiques (Nématodes et Diptères autres que Chironomidés).

\section{b) zone profonde}

Sur les 26 taxons recensés par les deux échantillonnages, 7 sont spécifiques aux sédiments, 11 aux substrats artificiels et seulement 8 sont communs.

Dans ce cas, les substrats artificiels attirent d'autant plus les organismes épibenthiques que l'habitat 
s'uniformise. En effet, les différences sont principalement dues aux Crustacés, aux Gastéropodes, aux Achètes et Oligochètes Naididae. II faut noter que dans les lacs du Jura, les Naididae (représentés sur les substrats artificiels essentiellement par l'espèce Stylaria lacustris n'ont jamais été recensés jusqu'ici à une profondeur inférieure à 10 mètres (C.E.M.A.G.R.E.F. in S.R.A.E. Fr. Comté, 1982-1987).

\subsubsection{Densité}

a) zone littorale

Nous avons récolté environ 13 fois plus d'individus par dixième de mètre carré sur les substrats que dans les sédiments (1334 individus pour 101).

La figure 7 présente les compositions des peuplements en densité relative.

$\mathrm{Ce}$ graphique montre que la composition du peuplement des substrats artificiels, quelle que soit la durée d'exposition, est très différente de celle des sédiments.

b) zone profonde

Nous avons récolté environ 1,5 fois plus d'individus par dixième de mètre carré sur les substrats que dans les sédiments (95 individus pour 65).

La figure 8 est construite de la même façon que la précédente.

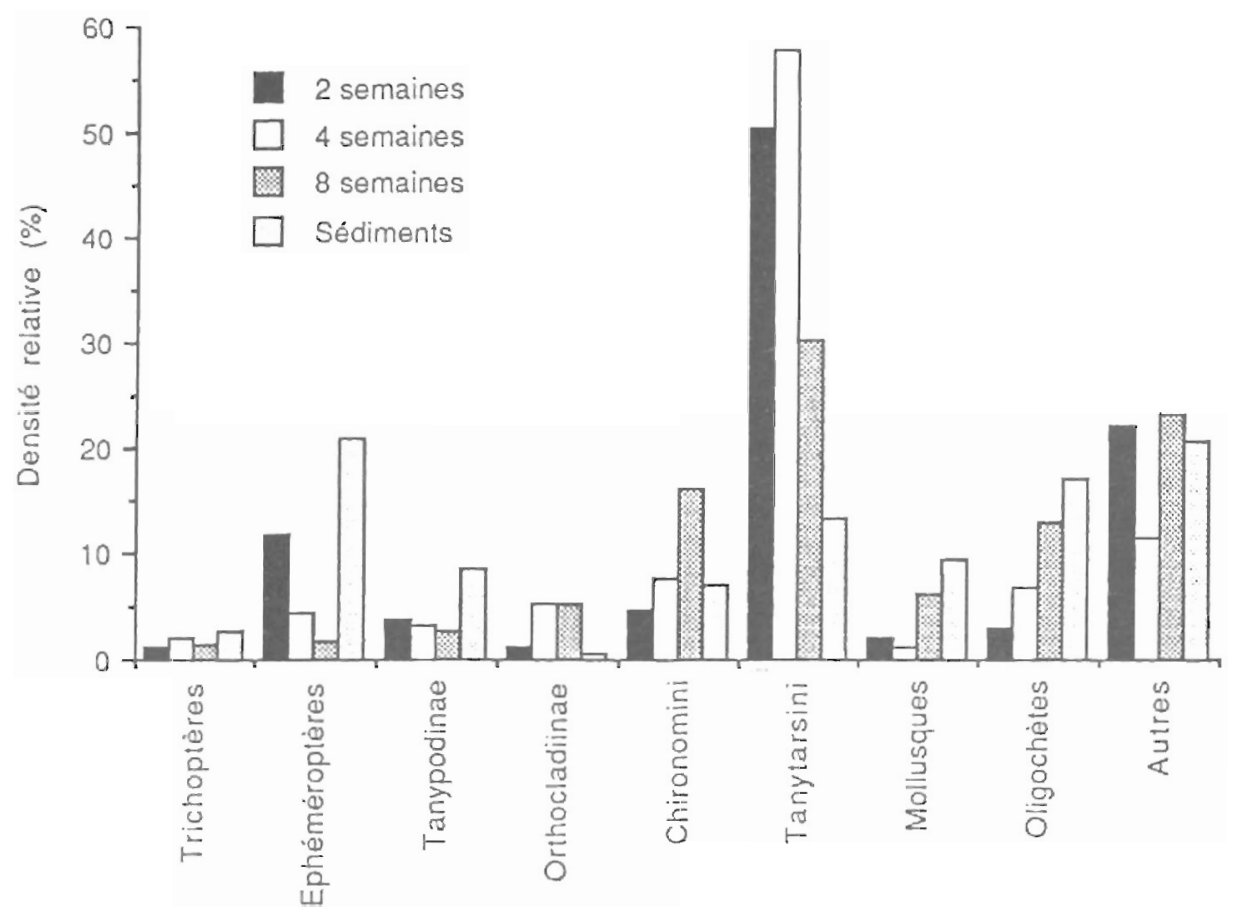

Fig. 7. - Comparaison des densités relatives en zone littorale.

Fig. 7. - Relatives densities comparison in the littoral zone. 


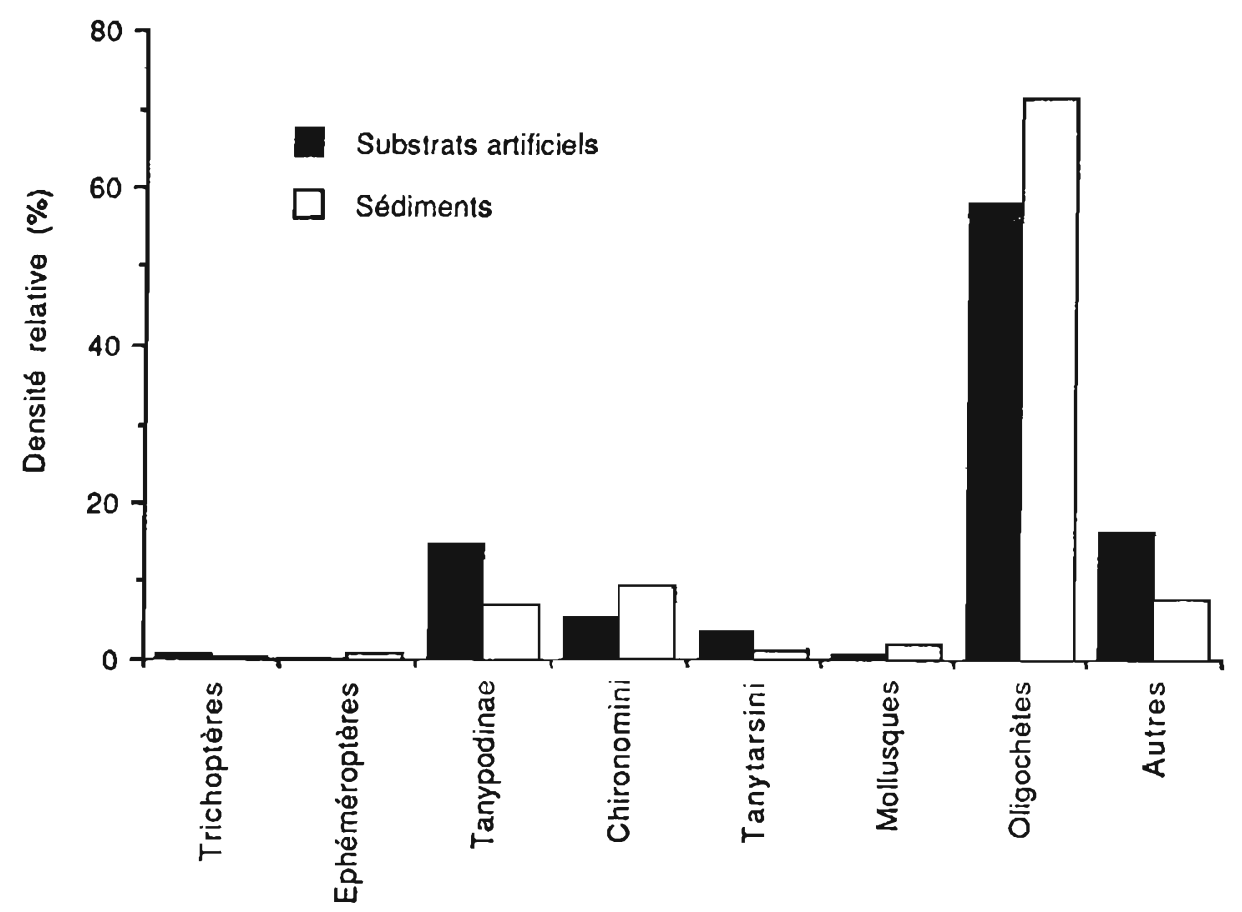

Fig. 8. - Comparaison des densités relatives en zone profonde.

Fig. 8. - Relatives densities comparison in the profundal zone.

Dans ce cas, la composition en densité relative semble similaire quel que soit le mode de prélèvement. II serait intéressant de savoir si la période de prélèvement de ces pièges (automne) et celle des sédiments (printemps) sont comparables au niveau de la composition générique du peuplement.

Ces résultats confirment que la durée d'exposition optimale est d'environ 4 semaines.

Par contre, quelle que soit la profondeur considérée, les deux peuplements sont sensiblement différents.
Cette faible similarité semble principalement due à la nature de la faune. En effet, les substrats artificiels présentent une gamme d'habitats beaucoup plus attractive que les sédiments, notamment pour l'épibenthos, et le phénomène s'accuse en zone profonde.

\section{Conclusions}

La variété taxonomique obtenue par l'utilisation des substrats artificiels exposés pendant 4 semaines en zone 
littorale est similaire à celle des sédiments. Toutefois, l'emploi des substrats artificiels est nettement avantageux sur le plan pratique (gain de temps et commodité de manipulation). Le tableau IV résume de façon synthétique les différences observées.
La figure 9 présente l'effort d'échantillonnage réalisé en zone littorale au moyen de chaque méthode (en centièmes de mètre carré) avec la variété et la densité obtenues conjointement (la durée d'exposition de 4 semaines étant considérée comme optimale).

Tableau IV. - Comparaison entre les deux protocoles (prélèvements de sédiments et substrats artificiels en variété taxonomique, effort d'échantillonnage et charge de travail). Table IV. - Comparison between the two protocols (sediments and artificial substrates sampling : taxonomic variety; sampling's effort; work's charge).

\begin{tabular}{|l|c|c|c|c|}
\cline { 2 - 5 } & Variété taxonomique & Effort d'échantillonnage & Temps de terrain & Temps de tri \\
\hline Sédiments $1,50 \mathrm{~m}$ & 47 & 42 litres & 1 journée & 10 joumées \\
& & $0,84 \mathrm{~m} 2$ & & \\
& $45 . A 1,50 \mathrm{~m}: 4 \mathrm{sem}$. & 2 substrats & 4 heures & 1 journéé \\
& $0,12 \mathrm{~m} 2$ & & \\
\hline Sédiments $30 \mathrm{~m}$ & 15 & 24 litres & 1 journée & 6 journées \\
& 19 & $0,48 \mathrm{~m} 2$ & & \\
S.A $30 \mathrm{~m}: 4 \mathrm{sem}$. & 15 & 2 substrats & 4 heures & 1 journée \\
& & $0,12 \mathrm{~m} 2$ & & \\
\hline
\end{tabular}

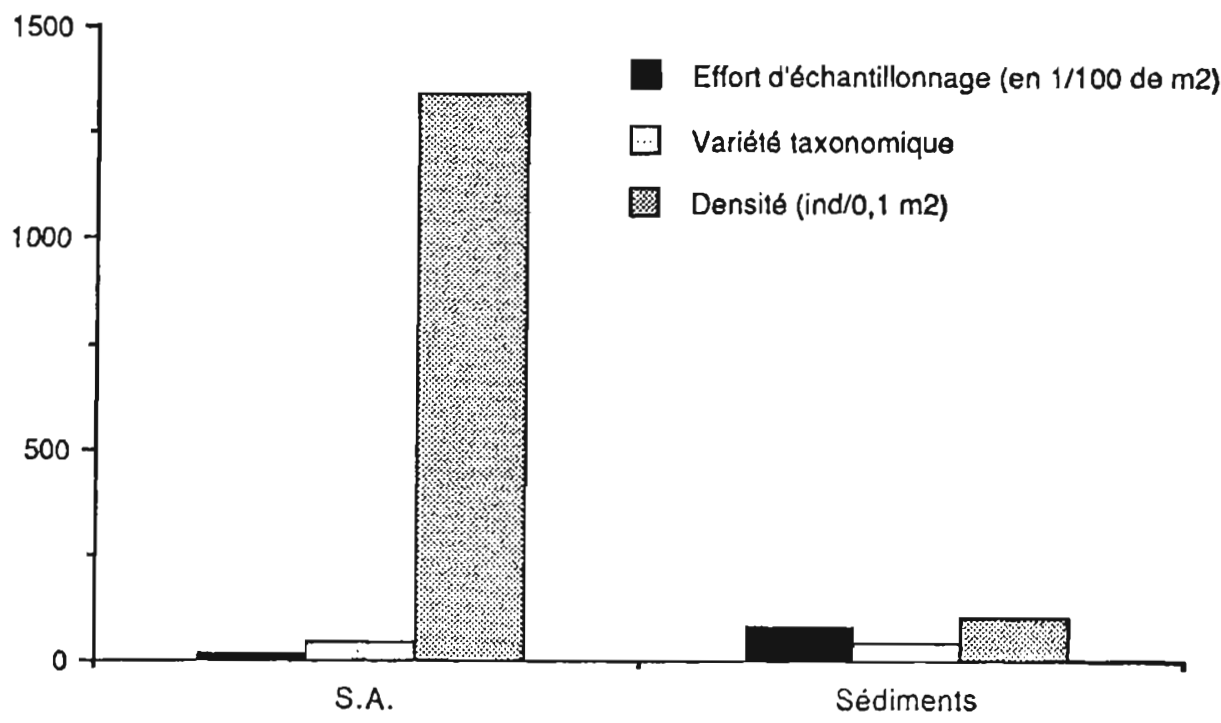

Fig. 9. - Résultats comparés entre les deux protocoles en fonction de l'effort d'échantillonnage. Fig. 9. - Compared results between the two protocols in relation to the sampling effort. 
Par contre, la densité et surtout la nature de la faune différent très sensiblement entre les deux méthodes; en effet, la faune recensée sur les substrats artificiels peut être qualifiée de plus épibenthique que celle des sédiments, quelle que soit la profondeur considérée :

- en zone littorale : prédominance des Orthocladiinae, des Cnidaires et des Coléoptères aux stades adulte et larvaire.

- en zone limnétique : présence de Naididae, de Tanypodinae, de Gammaridae et d'Asellidae en quantité importante.

Un protocole complet d'échantillonnage comparatif demeure à établir, en determinant la période de pose la plus favorable, et le nombre minimal de stations nécessaire à l'obtention d'une image stable de l'échantillon relatif au système.

Afin d'échapper à l'influence de la nature du substrat sur la composition des biocénoses benthiques, une méthode d'analyse biologique comparative fondée sur l'utilisation des substrats antificiels est préconisée.

En outre, il serait intéressant de coupler les substrats artificiels avec des pièges à émergence afin de capturer les nymphes et les imagos correspondant au peuplement larvaire des substrats artificiels permettant ainsi la constitution d'un répertoire taxonomique spécifique.

Enfin, l'utilisation de ce dispositif constitue un outil utile à l'approfondissement de l'écologie des espèces dans la mesure ou il permet de diffé- rencier les influences de l'habitat et celles de la qualité de l'eau dans leur distribution bathymétrique.

\section{BIBLIOGRAPHIE}

Anderson J.B. \& Mason W.T. 1968. A comparison of benthic macroinvertebrates collected by dredge and basket sampler. J. Water Poll. Central Fed., 40 (2) : 252-259.

Arthur J.W. \& Horning W.B. 1969. The use of artificial substrates in pollution surveys. Amer. Midi. Nat., 82 (1) : 83-99.

Benfield E., Hendricks A. \& Cairns J.Jr. 1974. Proficiences of two artificial substrates in collecting stream macroinvertebrates. Hydrobiologia, 45 (2) : 431-440.

Bishop J.E. 1972. Observations on the vertical distribution of the benthos in a Malaysian stream. Fresh. Biol., 3 : 147-156.

Bournaud M. \& Cellot B.. 1981. Méthodologie de prélèvement dans un fleuve en relation avec les mouvements de la faune benthique. Lab. Biol. Anim., Univ. Lyon I, 75 p.

Britt N.W. 1955. New methods for collecting bottom fauna from shoals or rubble bottoms of lakes and streams. Ecology, 36 : 214-526.

Cellot B. 1982. Cycle annuel et zonation de la dérive des macroinvertébrés du Rhône en amont de Lyon. Mém. thèse $3^{\text {e }}$ cycle, Univ. Lyon I, $160 \mathrm{p}$.

C.E.M.A.G.R.E.F. in S.R.A.E. Fr. Comté : Monographies écologiques des lacs du Jura:

- 1982 - Le lac de l'Abbaye: 61 p et annexes.

- 1984 - Les lacs de Clainaux: 99 p et annexes.

- 1985 - Les lacs de Maclu: 124 p et annexes

- 1986a - Lac d'llay: $95 \mathrm{p}$ et annexes

- 1986b - Lac de Châlain: I16 p et 
annexes

- 1987 - Lac de Bonlieu: 100 p et annexes

- 1987 - Lacs d'Etival : 87 p et annexes.

Coleman M. \& Hynes H.B.N. 1970. The vertical distribution of the invertebrate fauna in the bed of a stream. Limnol. Oceanogr., $15:$ 31-40.

Dickson K.L., Cairns J. Jr. \& Arnold J.C. 1971. An evaluation of the use of artificial basket-type substrates for sampling macrobenthic invertebrate organisms. Trans. Amer. Fish. Soc., 100 (9) : 553-559.

Dickson K.L. \& Cairns J. Jr. 1972. The relationship of freshwater macroinvertebrate communities collected by floating artificial subtrates to the Mac ArthurWilson equilibrum model. Amer. Midi. Natur., $88(1)$ : 68-75.

Egglishaw H.J. 1964. The distributional relationship between the bottom fauna and plant detritus in streams. J. Anim. Ecol., 33 : 463-476.

Fullner R.W. 1971. A comparison of macroinvertebrates collected by basket and modified multiplate samplers. J. Wat. Poll. Central Fed., 43 (3) : 494499.

Glime J.M. \& Clemons R.M. 1971. Species diversity of stream insects on Fontinalis sp. compared to diversity on artificial substrates. Ecology, 53 (3): 458-464.

Henson E.B. 1965. A cage sampler for collecting aquatic fauna. Turtox News, $\mathbf{4 3}$ (12) : 298-299.

Hester F.E. \& Denoy J.S. 1962. A multiple plate sampler for aquatic macroinvertebrates. Trans. Amer. Fish. Soc., 91 (4) : 420-421.

Hynes J.O. 1975. Downstream drift of invertebrates in a river in southern Ghana. Freshwat. Biol., $5:$ 515-532.

Jacobi G.Z. 1971. A quantitative antificial substrate sampler for benthic macroinvertebrates. Trans. Amer. Fish. Soc., 100 (1) : 136-138.
Kajak Z. 1964. Experimental investigations of benthos abundance in the bottom of Lake Sniardwy. Ekol. Polsk. A : 1231.

Khalaf G.1975. Utilisation de substrats artificiels en eau courante, pour l'étude de la répartition et de la dynamique de colonisation des macroinvertébrés benthiques. Mém. thèse $3^{\theta}$ cycle. Lyon : $91 \mathrm{p}$.

Laville H. 1974. Utilisation de substrats artificiels pour l'étude de la faune macrobenthique de la zone littorale rocheuse des lacs de montagne. Annls. Limnol., 10 (2) : 163-172.

Legendre L. \& Legendre P. 1979. Ecologie numérique. 2. La structure des données écologiques. Masson éd. Paris, $247 \mathrm{p}$.

Macan T.T. \& Kitching A. 1972. Some experiments with artificial substrates. Verh. Int. Verein. Limnol., 18: 213220.

Mc Daniel M.D. 1974. Design and preliminary evaluation of an improved artificial substrate sampler for aquatic macroinvertebrates. Prog. Fish. Cult, 36 (1) : 23-26.

Malesieux G.1976. Utilisation des substrats antificiels benthiques pour la détermination de la qualité des eaux. Mém. DUEHH, Lab. Hydrobiol., Fac. Sci. Univ. Fr. Comté, Besançon, 47 p.

Mason W.T., Weber C.I., Lewis Ph. A \& Julian E.C. 1973. Factors affecting the performance of basket and multiplate macroinvertebrate samplers. Freshwat. Biol., 3 : 409-436.

Moon H.P. 1935. Methods and apparatus suitable for investigation of stony littoral of oligotrophic lakes. Int. Rev. Ges. Hydrobiol. Hydrogr., 32 : 319-333.

Moon H.P. 1940. An investigation of the movements of freshwater invertebrate fauna. J. Anim. Ecol., 9 : 76-83.

Schwoerbel J. 1967. Das hyporheische interstitial als Grenzbiotop zwischen oberirdischem und subterranem Ökosystem und seine bedeutung für die 
primär-evolution von Kleinsthöhlenbewohnern. Arch. Hydrobiol., suppl. 33 : 1-62.

Scott D.C. 1958. Biological balance in streams. Sewage and Indust. Wastes, 30 (9) : 1169-1173.

Sents \& Braconnot E. 1966. Données écologiques sur la fixation d'invertébrés sur des plaques immergées. Int. Rev. Ges. Hydrobiol., 51 : 461-484.

Ulfstrand S., Nilson L.M. \& Stergar A. 1974. Composition and diversity of benthic species collectives colonizing implanted substrates in a south swedish stream. Ent. Scand., 5 : 115-122.
Verneaux J., Faessel B. \& Malesieux G. 1977. Note préliminaire à la proposition de nouvelles méthodes de détermination de la qualité des eaux courantes. Lab. Hydrobiol. Fac. Sci. Tech., Univ. Fr. Comté, Besançon.

Verneaux J., Verneaux V. \& Guyard A. 1993. Classification biologique des lacs jurassiens à l'aide d'une nouvelle méthode d'analyse des peuplements benthiques. I. Variété et densité de la faune. Annls Limnol. 29 (1) : 59-77.

Wene G. \& Wickliff E.L. 1940. Modification of a stream bottom and its effects on the fauna. California Entom., 72 : 131. 135. 